\title{
The human resource for health situation in Zambia: deficit and maldistribution
}

Paulo Ferrinho $^{*}$, Seter Siziya ${ }^{2}$, Fastone Goma² and Gilles Dussault ${ }^{1}$

\begin{abstract}
Introduction: Current health policy directions in Zambia are formulated in the National Health Strategic Plan. The Plan focuses on national health priorities, which include the human resources (HR) crisis. In this paper we describe the way the HRH establishment is distributed in the different provinces of Zambia, with a view to assess the dimension of shortages and of imbalances in the distribution of health workers by province and by level of care.

Population and methods: We used secondary data from the "March 2008 payroll data base", which lists all the public servants on the payroll of the Ministry of Health and of the National Health Service facilities. We computed rates and ratios and compared them.

Results: The highest relative concentration of all categories of workers was observed in Northern, Eastern, Lusaka, Western and Luapula provinces (in decreasing order of number of health workers).

The ratio of clinical officers (mid-level clinical practitioners) to general medical officer (doctors with university training) varied from 3.77 in the Lusaka to 19.33 in the Northwestern provinces. For registered nurses (3 to 4 years of mid-level training), the ratio went from 3.54 in the Western to 15.00 in Eastern provinces and for enrolled nurses (two years of basic training) from 4.91 in the Luapula to 36.18 in the Southern provinces.

This unequal distribution was reflected in the ratio of population per cadre. The provincial distribution of personnel showed a skewed staff distribution in favour of urbanized provinces, e.g. in Lusaka's doctor: population ratio was 1: 6,247 compared to Northern Province's ratio of 1: 65,763.

In the whole country, the data set showed only 109 staff in health posts: 1 clinical officer, 3 environmental health technologists, 2 registered nurses, 12 enrolled midwives, 32 enrolled nurses, and 59 other.

The vacancy rates for level 3 facilities(central hospitals, national level) varied from 5\% in Lusaka to 38\% in Copperbelt Province; for level 2 facilities (provincial level hospitals), from 30\% for Western to $70 \%$ for Copperbelt Province; for level 1 facilities (district level hospitals), from 54\% for the Southern to $80 \%$ for the Western provinces; for rural health centres, vacancies varied from 15\% to 63\% (for Lusaka and Luapula provinces respectively); for urban health centres the observed vacancy rates varied from 13\% for the Lusaka to $96 \%$ for the Western provinces. We observed significant shortages in most staff categories, except for support staff, which had a significant surplus.

Discussion and Conclusions: This case study documents how a peaceful, politically stable African country with a longstanding tradition of strategic management of the health sector and with a track record of innovative approaches dealt with its $\mathrm{HRH}$ problems, but still remains with a major absolute and relative shortage of health workers. The case of Zambia reinforces the idea that training more staff is necessary to address the human resources crisis, but it is not sufficient and has to be completed with measures to mitigate attrition and to increase productivity.
\end{abstract}

\footnotetext{
* Correspondence: pferrinho@ihmt.unl.pt

${ }^{1}$ International Public Health and Biostatistics Unit, CMDT, Instituto de Higiene

e Medicina Tropical, Universidade Nova de Lisboa and Associação para o

Desenvolvimento e Cooperação Garcia de Orta, Lisbon, Portugal

Full list of author information is available at the end of the article
} 


\section{Introduction}

This case study documents how a peaceful, politically stable African country with a longstanding tradition of strategic management of the health sector and with a track record of innovative approaches, dealt with its health workforce problems, but still faces a major absolute and relative deficit of health workers. We briefly describe the country context and we use official data from 2008 to analyse various dimensions of the health workforce, such as vacancies, attrition, and geographical imbalances. The case of Zambia reinforces the idea that training more staff is necessary to address the human resources crisis, but it is not sufficient and has to be completed with measures to mitigate attrition and to increase productivity.

\section{General Background}

Zambia's population was estimated at just under 12 million in 2007 by the United Nations. The country is divided into 9 provinces and 72 districts. It has one of the lowest Human Development Index (0.481, ranking 164 in the world), the second lowest for Southern Africa, after Mozambique (http://www.pnud.org.br/pobreza_desigualdade/reportagens/index.php?id01=3324\&lay=pde, accessed on 3 August 2009). It has one of the highest prevalence rates for HIV/AIDS in Africa (15.2\%) (http:// www.who.int/gho/countries/zmb/country_profiles/en/ index.html, accessed on 17 January 2011).

Current policy directions are formulated in the National Health Strategic Plan (NHSP 2006-2010) [1], the fourth of its kind. It presents a major departure from previous plans, in that it establishes national health priorities, which include addressing the human resources for health $(\mathrm{HRH})$ crisis $[2,3]$. The recognition of $\mathrm{HRH}$ as a priority derives from the estimation by the Ministry of Health $(\mathrm{MoH})$ that health services function with less than half of the health workers required to deliver basic health services [4].

In addition to the national health service (NHS) facilities, there is an emerging urban private-for-profit sector, plus private mine-based hospitals, and a not-for-profit private sector working in close partnership with the public services. At the time of the study, of the 1327 healthcare facilities in Zambia, $85 \%$ are government run facilities, $9 \%$ are private sector facilities and $6 \%$ are religious affiliated facilities. Most (99\%) of urban households reside within $5 \mathrm{~km}$ of a health facility compared to $50 \%$ of rural households [5].

There are six levels of care in the public sector and corresponding facilities (outreach services, health posts, health centres, and level 1- district, level-2 provincial and level-3 central hospitals).

Health Posts are intended to cater for populations of 500 households (3,500 people) in rural areas and 1,000 households (7,000 people) in the urban areas, or to be established within a $5 \mathrm{Km}$ radius for sparsely populated areas. The target is 3,000 health posts. In 2008, there were 171 health posts. They offer basic first aid rather than curative care.

Health Centres include Urban Health Centres, which are intended to serve a catchment population of 30,000 to 50,000 people, and Rural Health Centres, servicing a catchment area of $29 \mathrm{Km}$ radius or a population of 10,000. The target is 1,385. Totals of 1029 rural health centres and 265 urban health centres were recorded in 2008. For the purpose of defining approved prototype staff establishments, health centres are further subdivided into large and medium urban, zonal and medium rural $[6,7]$.

$1^{\text {st }}$ Level Referral Hospitals are found in 60 of the 72 districts and are intended to serve a population of between 80,000 and 200,000 with medical, surgical, obstetric and diagnostic services, including all clinical services to support health centre referrals. Currently, there are $721^{\text {st }}$ Level Referral Hospitals. There is an approved prototype staff establishment of 192 workers, common to all $1^{\text {st }}$ level hospitals [6,7].

$2^{\text {nd }}$ level referral, Provincial or General Hospitals are $2^{\text {nd }}$ level hospitals at provincial level and are intended to cater for a catchment population of 200,000 to 800,000 people, with services in internal medicine, general surgery, paediatrics, obstetrics and gynaecology, dental, psychiatry and intensive care services. There are 21 level 2 hospitals. These hospitals are also planned to act as referral centres for the $1^{\text {st }}$ level institutions, including the provision of technical back-up and training functions. There is need to rationalize the distribution of these facilities through right-sizing. For the purpose of defining approved prototype staff establishments, $2^{\text {nd }}$ level hospitals are further subdivided into urban (with a staff establishment of 629) and rural (with a staff establishment of 384) $[6,7]$.

$3^{\text {rd }}$ level or Central Hospitals are for catchment populations of 800,000 and above, and have sub-specializations in internal medicine, surgery, paediatrics, obstetrics, gynaecology, intensive care, psychiatry, training and research. These hospitals also act as referral centres for $2^{\text {nd }}$ level hospitals. Currently there are 6 such facilities in the country, of which 3 are in the Copperbelt Province. Again there is need to rationalize the distribution of these facilities [6,7].

Contractual arrangements with private providers, particularly the mission and mining sectors, are common $[6,7]$.

The National Health Service staff establishment covers these six types of facilities [8]. In this paper we describe the way this establishment is distributed in the different provinces of Zambia. 


\section{The Health Workforce: Stock and Distribution Population and methods}

Using the "March 2008 payroll data base", that lists all public servants on the payroll of $\mathrm{MoH}$ and of NHS facilities, we analysed data on the distribution of health workers by category and post, province, type of health facility and health care level. Figures on the number of inhabitants were obtained from the Zambia 2000 "census of population and housing", and extrapolated using expected growth rates for each province. Population figures for district level were not available.

The results of this analysis are explained in light of the literature available, and of findings from in-depth interviews by three of the authors (PF, SS \& FG) with key informants and personal observations carried out in the context of another parallel study (P Ferrinho, M Sidat, F Goma, G Dussault: Task-shifting - opinions and experiences of health workers in the Mozambican and Zambian National Health Services, submitted to Human Resour Health 2011).

\section{Results}

\section{Distribution of personnel across provinces}

The most numerous categories of health workers in all provinces are the Zambia Enrolled nurses, followed by Zambia Enrolled Midwives and Registered nurses. Variations between provinces observed at the level of health specific cadres are greater than at that of general support staff. The highest concentrations of health specific cadres are observed, in decreasing order, in the Central, Southern, Copperbelt and Northwestern provinces (Table 1).

Ratios of clinical officers, who are mid-level practitioners to general medical officer, who are physicians with university training, varied from 3.77 in Lusaka to 19.33 in Northwestern Province. For registered nurses (3 to 4 years of mid-level training), the ratio varied from 3.54 in Western to 15.00 in Eastern Province, and for Zambia enrolled nurses (two years of basic training) from 25.15 in Western Province to 115.67 in the Northwestern Province. The highest ratios for health specific cadres are observed for Zambia Enrolled nurses, followed by Zambia Enrolled Midwives and Registered nurses (Table 2).

There is a similar uneven distribution in the ratio of population per cadre (Table 3). For the 52 cadres listed, the best served provinces, were Copperbelt (13 cadres with a ratio above the national median), Southern (16), Lusaka (19), Central (23), Western (33 cadres), North-Western (36), Eastern (38), Luapula (40) and Northern (43).

The provincial distribution of health specific occupations showed a skewed staff distribution in favour of the most urbanized provinces (Lusaka and Copperbelt provinces). The Zambia enrolled nurse is the occupation with the most uniform distribution across provinces.

\section{Distribution of staff by levels of care}

Non-qualified health workers (ancillary staff) constituted the greatest majority of workers at Level 3 hospitals, followed by enrolled nurses and registered nurses. There were no consultant surgeons, anaesthetists, laboratory and radiology staff. For 50 occupational categories in the two provinces with Level 3 hospitals (Copperbelt and Lusaka), staffing levels were below the approved establishment for 26 and 35 categories respectively, above for 14 and 9 categories, and equal for 10 and 6 categories.

General medical officers represented between $0.3 \%$ and $2.6 \%$ of the workforce for Level 2 hospitals per province; clinical officers between $2 \%$ and $4 \%$; registered nurses between $4 \%$ and $8 \%$; enrolled nurses between $16 \%$ and $28 \%$ and general nonqualified workers between $41 \%$ and $57 \%$. The ratio of non-qualified workers to general medical officer varied from 19 to 137 . The ratio of all cadres per bed was generally low, and more so for general medical officers per 100 beds at between 0 and 4 .

For Level 1 hospitals the situation was similar. General medical officers represented between $0.3 \%$ to $2.9 \%$ of the total workforce; clinical officers between $2 \%$ to $6 \%$; registered nurses between $4 \%$ to $8 \%$; enrolled nurses between $17 \%$ to $34 \%$ and general non-qualified workers between $34 \%$ to $53 \%$; ratios to general medical officer varied from 16 to 159 . In Level 1 hospitals, the ratio of cadres per bed was also low: the ratio of general medical officers per 100 beds varied between 0 and 3 .

Only two physicians worked in rural health centres in the whole country. Placing doctors at this level may be questionable, but some large health centres function as first level hospitals without being categorized as such by the $\mathrm{MoH}$, and would therefore justify employing physicians. Non-qualified workers formed between 31\% to 54\% of all staff in rural health centres; clinical officers between $3 \%$ and $11 \%$; enrolled midwives between $3 \%$ and $14 \%$; environmental health technologist between $8 \%$ and $15 \%$; and enrolled nurses between $16 \%$ and $27 \%$. The ratio of non-qualified workers to clinical officer varied between 3 and 16 per province.

Urban health centres employed 17 doctors. These facilities also often functioned as first level hospitals, especially in Lusaka which had only tertiary hospitals. The infrastructure of urban health centres was upgraded to enable them to function at a higher level of service provision. Non-qualified workers constitute between $17 \%$ to $33 \%$ of total staff; clinical officers between $4 \%$ and $11 \%$; enrolled midwives between $9 \%$ and $2 \%$; environmental health technologist between $0 \%$ and $7 \%$; and enrolled nurses between $22 \%$ and $45 \%$. The ratio of non-qualified workers to clinical officer varied between 2 and 8 by province.

In the entire country, the data set reported a total 109 staff in health posts: 1 clinical officer, 3 environmental 
Table 1 Distribution of health workers (\% of total workers on NHS payroll) by province

\begin{tabular}{|c|c|c|c|c|c|c|c|c|c|}
\hline Occupation & Central & Copperbelt & Eastern & Luapula & Lusaka & North-Western & Northern & Southern & Western \\
\hline & Total $=1787$ & Total $=4987$ & Total $=2281$ & Total $=1123$ & Total $=5617$ & Total $=1190$ & Total $=2153$ & Total $=3533$ & Total $=1423$ \\
\hline & Percent & Percent & Percent & Percent & Percent & Percent & Percent & Percent & Percent \\
\hline General Medical Officer & 0,62 & 0,62 & 0,26 & 0,98 & 1,09 & 0,25 & 0,42 & 0,31 & 0,91 \\
\hline Anaesthesiologist & 0,00 & 0,00 & 0,00 & 0,09 & 0,00 & 0,00 & 0,00 & 0,00 & 0,00 \\
\hline Biomedical Scientist & 0,06 & 0,10 & 0,00 & 0,00 & 0,37 & 0,00 & 0,05 & 0,11 & 0,00 \\
\hline Clinical Officer & 5,99 & 4,31 & 4,12 & 4,36 & 4,09 & 4,87 & 4,37 & 5,26 & 5,41 \\
\hline Clinical Officer Anaesthesia & 0,28 & 0,14 & 0,13 & 0,27 & 0,18 & 0,25 & 0,23 & 0,37 & 0,07 \\
\hline Clinical Officer Dental & 0,11 & 0,04 & 0,04 & 0,00 & 0,07 & 0,00 & 0,00 & 0,08 & 0,07 \\
\hline Clinical Officer Dermatology & 0,06 & 0,04 & 0,00 & 0,00 & 0,00 & 0,17 & 0,05 & 0,00 & 0,07 \\
\hline Clinical Officer Eye, Nose, and Throat & 0,00 & 0,02 & 0,00 & 0,00 & 0,00 & 0,00 & 0,00 & 0,00 & 0,00 \\
\hline Clinical Officer Ophthalmology & 0,00 & 0,04 & 0,04 & 0,00 & 0,05 & 0,08 & 0,05 & 0,06 & 0,28 \\
\hline Clinical Officer TB, HIV/AIDS & 0,00 & 0,00 & 0,00 & 0,00 & 0,00 & 0,00 & 0,00 & 0,03 & 0,00 \\
\hline Clinical Officer Psychiatry & 0,11 & 0,04 & 0,26 & 0,00 & 0,05 & 0,00 & 0,05 & 0,08 & 0,00 \\
\hline Consultant & 0,11 & 0,28 & 0,13 & 0,18 & 0,84 & 0,00 & 0,00 & 0,06 & 0,07 \\
\hline Consultant Anaesthesia & 0,00 & 0,02 & 0,00 & 0,00 & 0,00 & 0,08 & 0,00 & 0,00 & 0,00 \\
\hline Consultant Obstetrics and Gynaecologist & 0,06 & 0,00 & 0,00 & 0,09 & 0,00 & 0,08 & 0,00 & 0,03 & 0,00 \\
\hline Consultant Ophthalmology & 0,00 & 0,02 & 0,00 & 0,00 & 0,00 & 0,00 & 0,00 & 0,00 & 0,00 \\
\hline Consultant Surgeon & 0,00 & 0,02 & 0,00 & 0,00 & 0,00 & 0,00 & 0,00 & 0,00 & 0,00 \\
\hline Dental Surgeon & 0,06 & 0,06 & 0,04 & 0,00 & 0,07 & 0,08 & 0,05 & 0,00 & 0,07 \\
\hline Dental Technician & 0,06 & 0,00 & 0,00 & 0,00 & 0,00 & 0,00 & 0,00 & 0,00 & 0,00 \\
\hline Dental Technologist & 0,11 & 0,12 & 0,22 & 0,09 & 0,05 & 0,08 & 0,05 & 0,14 & 0,07 \\
\hline Dental Therapist & 0,78 & 0,58 & 0,44 & 0,36 & 0,61 & 0,34 & 0,37 & 0,48 & 0,49 \\
\hline Environmental Health Expert & 0,22 & 0,22 & 0,13 & 0,36 & 0,05 & 0,50 & 0,28 & 0,23 & 0,07 \\
\hline Environmental Health Officer & 0,22 & 0,12 & 0,35 & 0,45 & 0,14 & 0,17 & 0,42 & 0,23 & 0,35 \\
\hline Environmental Health Technologist & 4,92 & 1,82 & 4,56 & 3,74 & 1,51 & 4,37 & 3,99 & 4,70 & 6,25 \\
\hline Laboratory Technologist & 1,12 & 0,94 & 0,53 & 0,98 & 0,66 & 0,76 & 0,56 & 0,76 & 0,63 \\
\hline Laboratory Technician & 0,95 & 1,16 & 0,66 & 0,98 & 0,64 & 0,84 & 0,65 & 0,82 & 0,70 \\
\hline Medical Licentiate & 0,11 & 0,02 & 0,26 & 0,18 & 0,07 & 0,08 & 0,14 & 0,03 & 0,21 \\
\hline Nutritionist & 0,28 & 0,18 & 0,39 & 0,18 & 0,28 & 0,17 & 0,23 & 0,25 & 0,14 \\
\hline Occupational Health Technologist & 0,00 & 0,00 & 0,00 & 0,00 & 0,00 & 0,00 & 0,00 & 0,03 & 0,00 \\
\hline Occupational Therapist & 0,00 & 0,00 & 0,00 & 0,00 & 0,00 & 0,00 & 0,00 & 0,03 & 0,00 \\
\hline Pharmacist & 0,28 & 0,24 & 0,13 & 0,09 & 0,16 & 0,00 & 0,14 & 0,20 & 0,14 \\
\hline Pharmacy Dispenser & 1,68 & 1,46 & 0,79 & 0,71 & 1,10 & 0,50 & 0,74 & 1,25 & 1,48 \\
\hline Pharmacy Technician & 0,11 & 0,12 & 0,04 & 0,27 & 0,00 & 0,17 & 0,05 & 0,03 & 0,14 \\
\hline Pharmacy Technologist & 0,28 & 0,42 & 0,53 & 0,53 & 0,39 & 0,34 & 0,19 & 0,42 & 0,14 \\
\hline
\end{tabular}


Table 1 Distribution of health workers (\% of total workers on NHS payroll) by province (Continued)

\begin{tabular}{|c|c|c|c|c|c|c|c|c|c|}
\hline Physiotherapist & 0,95 & 0,80 & 0,35 & 0,45 & 0,62 & 0,42 & 0,37 & 0,57 & 0,56 \\
\hline Physiotherapy Technologist & 0,06 & 0,04 & 0,18 & 0,27 & 0,04 & 0,00 & 0,14 & 0,20 & 0,07 \\
\hline Public Health Nurse & 0,11 & 0,04 & 0,04 & 0,00 & 0,14 & 0,08 & 0,05 & 0,00 & 0,21 \\
\hline Radiographer & 0,78 & 0,78 & 0,53 & 0,36 & 0,64 & 0,34 & 0,46 & 0,79 & 0,07 \\
\hline Radiography Technologist & 0,00 & 0,00 & 0,00 & 0,00 & 0,07 & 0,00 & 0,19 & 0,00 & 0,00 \\
\hline Registered ICU Nurse & 0,00 & 0,00 & 0,04 & 0,00 & 0,00 & 0,00 & 0,00 & 0,03 & 0,00 \\
\hline Registered Midwife & 2,13 & 1,95 & 0,70 & 0,53 & 1,80 & 1,18 & 0,74 & 1,33 & 0,84 \\
\hline Registered Nurse & 5,60 & 6,36 & 3,95 & 4,81 & 6,48 & 3,36 & 5,57 & 4,02 & 3,23 \\
\hline Registered Psychiatry Nurse & 0,11 & 0,02 & 0,00 & 0,00 & 0,27 & 0,00 & 0,00 & 0,06 & 0,00 \\
\hline Registered Theatre Nurse & 0,28 & 0,22 & 0,22 & 0,36 & 0,32 & 0,08 & 0,19 & 0,20 & 0,14 \\
\hline Registrar & 0,06 & 0,48 & 0,04 & 0,27 & 0,80 & 0,00 & 0,00 & 0,14 & 0,00 \\
\hline Registered Nurse Ophthalmology & 0,00 & 0,00 & 0,00 & 0,00 & 0,02 & 0,00 & 0,00 & 0,00 & 0,00 \\
\hline Resident Medical Officer & 0,73 & 1,44 & 0,70 & 0,80 & 1,57 & 1,26 & 0,65 & 1,02 & 0,77 \\
\hline Zambia Enrolled Midwife & 9,85 & 7,62 & 7,85 & 4,81 & 5,38 & 3,70 & 6,60 & 11,27 & 5,55 \\
\hline Zambia Enrolled Nurse & 31,45 & 20,93 & 20,12 & 24,93 & 18,23 & 29,16 & 16,40 & 22,90 & 22,98 \\
\hline Zambia Enrolled Ophthalmology & 0,00 & 0,00 & 0,00 & 0,00 & 0,00 & 0,00 & 0,00 & 0,03 & 0,00 \\
\hline Zambia Enrolled Psychiatry Nurse & 0,22 & 0,16 & 0,09 & 0,45 & 0,73 & 0,00 & 0,05 & 0,03 & 0,14 \\
\hline Zambia Enrolled Theatre Nurse & 0,17 & 0,14 & 0,39 & 0,18 & 0,05 & 0,00 & 0,19 & 0,14 & 0,21 \\
\hline Other & 28,93 & 45,84 & 50,72 & 46,93 & 50,35 & 46,22 & 55,36 & 41,30 & 47,43 \\
\hline
\end{tabular}

Source: MoH, March 2008 payroll data base 
Table 2 Ratio of different occupations per General Medical Officer per province

\begin{tabular}{|c|c|c|c|c|c|c|c|c|c|}
\hline Occupation & Central & Copperbelt & Eastern & Luapula & Lusaka & North-Western & Northern & Southern & Western \\
\hline & ratio & ratio & ratio & ratio & ratio & ratio & ratio & ratio & ratio \\
\hline General Medical Officer & 1,00 & 1,00 & 1,00 & 1,00 & 1,00 & 1,00 & 1,00 & 1,00 & 1,00 \\
\hline Anaesthesiologist & 0,00 & 0,00 & 0,00 & 0,09 & 0,00 & 0,00 & 0,00 & 0,00 & 0,00 \\
\hline Biomedical Scientist & 0,09 & 0,16 & 0,00 & 0,00 & 0,34 & 0,00 & 0,11 & 0,36 & 0,00 \\
\hline Clinical Officer & 9,73 & 6,94 & 15,67 & 4,45 & 3,77 & 19,33 & 10,44 & 16,91 & 5,92 \\
\hline Clinical Officer Anaesthesia & 0,45 & 0,23 & 0,50 & 0,27 & 0,16 & 1,00 & 0,56 & 1,18 & 0,08 \\
\hline Clinical Officer Dental & 0,18 & 0,06 & 0,17 & 0,00 & 0,07 & 0,00 & 0,00 & 0,27 & 0,08 \\
\hline Clinical Officer Dermatology & 0,09 & 0,06 & 0,00 & 0,00 & 0,00 & 0,67 & 0,11 & 0,00 & 0,08 \\
\hline Clinical Officer Eye, Nose, and Throat & 0,00 & 0,03 & 0,00 & 0,00 & 0,00 & 0,00 & 0,00 & 0,00 & 0,00 \\
\hline Clinical Officer Ophthalmology & 0,00 & 0,06 & 0,17 & 0,00 & 0,05 & 0,33 & 0,11 & 0,18 & 0,31 \\
\hline Clinical Officer TB, HIV/AIDS & 0,00 & 0,00 & 0,00 & 0,00 & 0,00 & 0,00 & 0,00 & 0,09 & 0,00 \\
\hline Clinical Officer Psychiatry & 0,18 & 0,06 & 1,00 & 0,00 & 0,05 & 0,00 & 0,11 & 0,27 & 0,00 \\
\hline Consultant & 0,18 & 0,45 & 0,50 & 0,18 & 0,77 & 0,00 & 0,00 & 0,18 & 0,08 \\
\hline Consultant Anaesthesia & 0,00 & 0,03 & 0,00 & 0,00 & 0,00 & 0,33 & 0,00 & 0,00 & 0,00 \\
\hline Consultant Obstetrics and Gynaecologist & 0,09 & 0,00 & 0,00 & 0,09 & 0,00 & 0,33 & 0,00 & 0,09 & 0,00 \\
\hline Consultant Ophthalmology & 0,00 & 0,03 & 0,00 & 0,00 & 0,00 & 0,00 & 0,00 & 0,00 & 0,00 \\
\hline Consultant Surgeon & 0,00 & 0,03 & 0,00 & 0,00 & 0,00 & 0,00 & 0,00 & 0,00 & 0,00 \\
\hline Dental Surgeon & 0,09 & 0,10 & 0,17 & 0,00 & 0,07 & 0,33 & 0,11 & 0,00 & 0,08 \\
\hline Dental Technician & 0,09 & 0,00 & 0,00 & 0,00 & 0,00 & 0,00 & 0,00 & 0,00 & 0,00 \\
\hline Dental Technologist & 0,18 & 0,19 & 0,83 & 0,09 & 0,05 & 0,33 & 0,11 & 0,45 & 0,08 \\
\hline Dental Therapist & 1,27 & 0,94 & 1,67 & 0,36 & 0,56 & 1,33 & 0,89 & 1,55 & 0,54 \\
\hline Environmental Health Expert & 0,36 & 0,35 & 0,50 & 0,36 & 0,05 & 2,00 & 0,67 & 0,73 & 0,08 \\
\hline Environmental Health Officer & 0,36 & 0,19 & 1,33 & 0,45 & 0,13 & 0,67 & 1,00 & 0,73 & 0,38 \\
\hline Environmental Health Technologist & 8,00 & 2,94 & 17,33 & 3,82 & 1,39 & 17,33 & 9,56 & 15,09 & 6,85 \\
\hline Laboratory Technologist & 1,82 & 1,52 & 2,00 & 1,00 & 0,61 & 3,00 & 1,33 & 2,45 & 0,69 \\
\hline Laboratory Technician & 1,55 & 1,87 & 2,50 & 1,00 & 0,59 & 3,33 & 1,56 & 2,64 & 0,77 \\
\hline Medical Licentiate & 0,18 & 0,03 & 1,00 & 0,18 & 0,07 & 0,33 & 0,33 & 0,09 & 0,23 \\
\hline Nutritionist & 0,45 & 0,29 & 1,50 & 0,18 & 0,26 & 0,67 & 0,56 & 0,82 & 0,15 \\
\hline Occupational Health Technologist & 0,00 & 0,00 & 0,00 & 0,00 & 0,00 & 0,00 & 0,00 & 0,09 & 0,00 \\
\hline Occupational Therapist & 0,00 & 0,00 & 0,00 & 0,00 & 0,00 & 0,00 & 0,00 & 0,09 & 0,00 \\
\hline Pharmacist & 0,45 & 0,39 & 0,50 & 0,09 & 0,15 & 0,00 & 0,33 & 0,64 & 0,15 \\
\hline Pharmacy Dispenser & 2,73 & 2,35 & 3,00 & 0,73 & 1,02 & 2,00 & 1,78 & 4,00 & 1,62 \\
\hline Pharmacy Technician & 0,18 & 0,19 & 0,17 & 0,27 & 0,00 & 0,67 & 0,11 & 0,09 & 0,15 \\
\hline Pharmacy Technologist & 0,45 & 0,68 & 2,00 & 0,55 & 0,36 & 1,33 & 0,44 & 1,36 & 0,15 \\
\hline Physiotherapist & 1,55 & 1,29 & 1,33 & 0,45 & 0,57 & 1,67 & 0,89 & 1,82 & 0,62 \\
\hline Physiotherapy Technologist & 0,09 & 0,06 & 0,67 & 0,27 & 0,03 & 0,00 & 0,33 & 0,64 & 0,08 \\
\hline Public Health Nurse & 0,18 & 0,06 & 0,17 & 0,00 & 0,13 & 0,33 & 0,11 & 0,00 & 0,23 \\
\hline Radiographer & 1,27 & 1,26 & 2,00 & 0,36 & 0,59 & 1,33 & 1,11 & 2,55 & 0,08 \\
\hline Radiography Technologist & 0,00 & 0,00 & 0,00 & 0,00 & 0,07 & 0,00 & 0,44 & 0,00 & 0,00 \\
\hline Registered ICU Nurse & 0,00 & 0,00 & 0,17 & 0,00 & 0,00 & 0,00 & 0,00 & 0,09 & 0,00 \\
\hline Registered Midwife & 3,45 & 3,13 & 2,67 & 0,55 & 1,66 & 4,67 & 1,78 & 4,27 & 0,92 \\
\hline Registered Nurse & 9,09 & 10,23 & 15,00 & 4,91 & 5,97 & 13,33 & 13,33 & 12,91 & 3,54 \\
\hline Registered Psychiatry Nurse & 0,18 & 0,03 & 0,00 & 0,00 & 0,25 & 0,00 & 0,00 & 0,18 & 0,00 \\
\hline Registered Theatre Nurse & 0,45 & 0,35 & 0,83 & 0,36 & 0,30 & 0,33 & 0,44 & 0,64 & 0,15 \\
\hline Registrar & 0,09 & 0,77 & 0,17 & 0,27 & 0,74 & 0,00 & 0,00 & 0,45 & 0,00 \\
\hline Registered Nurse Ophthalmology & 0,00 & 0,00 & 0,00 & 0,00 & 0,02 & 0,00 & 0,00 & 0,00 & 0,00 \\
\hline Resident Medical Officer & 1,18 & 2,32 & 2,67 & 0,82 & 1,44 & 5,00 & 1,56 & 3,27 & 0,85 \\
\hline
\end{tabular}


Table 2 Ratio of different occupations per General Medical Officer per province (Continued)

\begin{tabular}{cccccccccc}
\hline \hline Zambia Enrolled Midwife & 16,00 & 12,26 & 29,83 & 4,91 & 4,95 & 14,67 & 15,78 & 36,18 & 6,08 \\
\hline Zambia Enrolled Nurse & 51,09 & 33,68 & 76,50 & 25,45 & 16,79 & 115,67 & 39,22 & 73,55 & 25,15 \\
\hline Zambia Enrolled Ophthalmology & 0,00 & 0,00 & 0,00 & 0,00 & 0,00 & 0,00 & 0,00 & 0,09 & 0,00 \\
\hline Zambia Enrolled Psychiatry Nurse & 0,36 & 0,26 & 0,33 & 0,45 & 0,67 & 0,00 & 0,11 & 0,09 & 0,15 \\
\hline Zambia Enrolled Theatre Nurse & 0,27 & 0,23 & 1,50 & 0,18 & 0,05 & 0,00 & 0,44 & 0,45 & 0,23 \\
\hline Other & 47,00 & 73,74 & 192,83 & 47,91 & 46,36 & 183,33 & 132,44 & 132,64 & 51,92 \\
\hline
\end{tabular}

Table 3 Population per health worker per province, Zambia, 2008

\begin{tabular}{|c|c|c|c|c|c|c|c|c|c|}
\hline Position & Central & Copperbelt & Eastern & Luapula & Lusaka & N Western & Northern & Southern & Western \\
\hline & ratio & ratio & ratio & ratio & ratio & ratio & ratio & ratio & ratio \\
\hline General Medical Officer & 111648 & 53933 & 249819 & 87875 & 29019 & 237528 & 173177 & 129206 & 66681 \\
\hline Anaesthesiologist & - & - & - & 966624 & - & - & - & - & - \\
\hline Biomedical Scientist & 1228125 & 334385 & - & - & 84293 & - & 1558589 & 355318 & - \\
\hline Clinical Officer & 11478 & 7776 & 15946 & 19727 & 7696 & 12286 & 16581 & 7641 & 11258 \\
\hline Clinical Officer Anaesthesia & 245625 & 238846 & 499637 & 322208 & 177016 & 237528 & 311718 & 109328 & 866854 \\
\hline Clinical Officer Dental & 614063 & 835962 & 1498912 & - & 442540 & - & - & 473757 & 866854 \\
\hline Clinical Officer Dermatology & 1228125 & 835962 & - & - & - & 356293 & 1558589 & - & 866854 \\
\hline Clinical Officer Eye, Nose, and Throat & - & 1671923 & - & - & - & - & - & - & - \\
\hline Clinical Officer Ophthalmology & - & 835962 & 1498912 & - & 590053 & 712585 & 1558589 & 710635 & 216714 \\
\hline Clinical Officer TB, HIV/AIDS & - & - & - & - & - & - & - & 1421270 & - \\
\hline clinical Officer Psychiatry & 614063 & 835962 & 249819 & - & 590053 & - & 1558589 & 473757 & - \\
\hline Consultant & 614063 & 119423 & 499637 & 483312 & 37663 & - & - & 710635 & 866854 \\
\hline Consultant Anaesthesia & - & 1671923 & - & - & - & 712585 & - & - & - \\
\hline Consultant Obstetrics and Gynaecologist & 1228125 & - & - & 966624 & - & 712585 & - & 1421270 & - \\
\hline Consultant Ophthalmology & - & 1671923 & - & - & - & - & - & - & - \\
\hline Consultant Surgeon & - & 1671923 & - & - & - & - & - & - & - \\
\hline Dental Surgeon & 1228125 & 557308 & 1498912 & - & 442540 & 712585 & 1558589 & - & 866854 \\
\hline Dental Technician & 1228125 & - & - & - & - & - & - & - & - \\
\hline Dental Technologist & 614063 & 278654 & 299782 & 966624 & 590053 & 712585 & 1558589 & 284254 & 866854 \\
\hline Dental Therapist & 87723 & 57653 & 149891 & 241656 & 52064 & 178146 & 194824 & 83604 & 123836 \\
\hline Environmental Health Expert & 307031 & 151993 & 499637 & 241656 & 590053 & 118764 & 259765 & 177659 & 866854 \\
\hline Environmental Health Officer & 307031 & 278654 & 187364 & 193325 & 221270 & 356293 & 173177 & 177659 & 173371 \\
\hline Environmental Health Technologist & 13956 & 18373 & 14413 & 23015 & 20825 & 13704 & 18123 & 8562 & 9740 \\
\hline Laboratory Technologist & 61406 & 35573 & 124909 & 87875 & 47842 & 79176 & 129882 & 52640 & 96317 \\
\hline Laboratory Technician & 72243 & 28826 & 99927 & 87875 & 49171 & 71259 & 111328 & 49009 & 86685 \\
\hline Medical Licentiate & 614063 & 1671923 & 249819 & 483312 & 442540 & 712585 & 519530 & 1421270 & 288951 \\
\hline Nutritionist & 245625 & 185769 & 166546 & 483312 & 110635 & 356293 & 311718 & 157919 & 433427 \\
\hline Occupational Health Technologist & - & - & - & - & - & - & - & 1421270 & - \\
\hline Occupational Therapist & - & - & - & - & - & - & - & 1421270 & - \\
\hline Pharmacist & 245625 & 139327 & 499637 & 966624 & 196684 & - & 519530 & 203039 & 433427 \\
\hline Pharmacy Dispenser & 40938 & 22903 & 83273 & 120828 & 28551 & 118764 & 97412 & 32302 & 41279 \\
\hline Pharmacy Technician & 614063 & 278654 & 1498912 & 322208 & - & 356293 & 1558589 & 1421270 & 433427 \\
\hline Pharmacy Technologist & 245625 & 79615 & 124909 & 161104 & 80462 & 178146 & 389647 & 94751 & 433427 \\
\hline Physiotherapist & 72243 & 41798 & 187364 & 193325 & 50576 & 142517 & 194824 & 71064 & 108357 \\
\hline Physiotherapy Technologist & 1228125 & 835962 & 374728 & 322208 & 885080 & - & 519530 & 203039 & 866854 \\
\hline Public Health Nurse & 614063 & 835962 & 1498912 & - & 221270 & 712585 & 1558589 & - & 288951 \\
\hline
\end{tabular}


Table 3 Population per health worker per province, Zambia, 2008 (Continued)

\begin{tabular}{cccccccccc}
\hline Radiographer & 87723 & 42870 & 124909 & 241656 & 49171 & 178146 & 155859 & 50760 & 866854 \\
\hline Radiography Technologist & - & - & - & - & 442540 & - & 389647 & - \\
\hline Registered ICU Nurse & - & - & 1498912 & - & - & - & - & 1421270 & - \\
\hline Registered Midwife & 32319 & 17236 & 93682 & 161104 & 17526 & 50899 & 97412 & 30240 & 72238 \\
\hline Registered Nurse & 12281 & 5274 & 16655 & 17900 & 4863 & 17815 & 12988 & 10009 & 18845 \\
\hline Registered Psychiatry Nurse & 614063 & 1671923 & - & - & 118011 & - & - & 710635 & - \\
\hline Registered Theatre Nurse & 245625 & 151993 & 299782 & 241656 & 98342 & 712585 & 389647 & 203039 & 433427 \\
\hline Registrar & 1228125 & 69663 & 1498912 & 322208 & 39337 & - & - & 284254 \\
\hline Registered Nurse Ophthalmology & - & - & - & - & 1770159 & - & - & - \\
\hline Resident Medical Officer & 94471 & 23221 & 93682 & 107403 & 20115 & 47506 & 111328 & 39480 & 78805 \\
\hline Zambia Enrolled Midwife & 6978 & 4400 & 8374 & 17900 & 5861 & 16195 & 10976 & 3571 & 10973 \\
\hline Zambia Enrolled Nurse & 2185 & 1601 & 3266 & 3452 & 1729 & 2054 & 4415 & 1757 & 2651 \\
\hline Zambia Enrolled Ophthalmology & - & - & - & - & - & - & - & 1421270 & - \\
\hline Zambia Enrolled Psychiatry Nurse & 307031 & 208990 & 749456 & 193325 & 43175 & - & 1558589 & 1421270 & 433427 \\
\hline Zambia Enrolled Theatre Nurse & 409375 & 238846 & 166546 & 483312 & 590053 & - & 389647 & 284254 & 288951 \\
\hline General support staff & 2375 & 731 & 1296 & 1834 & 626 & 1296 & 1308 & 974 & 1284 \\
\hline
\end{tabular}

Sources: population figures were obtained from the Zambia 2000 "census of population and housing", and extrapolated using expected growth rates for each province; for health workers the source was the $\mathrm{MoH}$, March 2008 payroll database

health technologists, 2 registered nurses, 12 enrolled midwives, 32 enrolled nurses, and 59 others.

\section{Vacancy rates}

For Level 3 facilities, vacancy rates varied between 38\% in the Copperbelt Province and 5\% in the Lusaka Province; for Level 2 facilities, figures were $30 \%$ and $70 \%$ in the Western and Copperbelt provinces; for Level 1 facilities, $54 \%$ and $80 \%$ for the Southern and Western provinces. For rural health centres, rates varied between $15 \%$ and 63\% (Lusaka and Luapula) and for urban health centres between $13 \%$ for Lusaka and $96 \%$ for the Western provinces.

\section{Discussion: explaining the observed shortages and maldistribution}

Zambia enrolled nurses are the most prevalent health specific cadre. This cannot be ignored in any policy to correct the impact of shortages and imbalances.

We identified a severe shortage, reflected in high vacancy rates of personnel in Zambia, associated with imbalances between provinces, levels of care and in the mix of cadres.

Shortages might be due to the inability to compensate for attrition and growing needs with scaled-up production, recruitment and retention of health workers.

High vacancy rates have been described in other studies: Picazo and Kagulura report that the percentage of vacant posts in $2005 / 2006$ was $42 \%$ in rural health centres, $22 \%$ in urban health centres, and $41 \%$ in hospitals (or 33.6\% overall). Key posts left vacant all involved professional staff. Districts with high rates of vacancy (> 50\%) among professional staff included: Chilubi, 79\%; Chinsali, 58\%;
Kalomo, 59\%; Kasama, 66\%; Mpika, 57\%; Mpongwe, 53\%; Mufulira, 66\%; Nakonde, 60\%; Namwala, 54\%; Sesheka, 74\%; Shangombo, 56\% [9].

Geographical imbalances of personnel can be attributable to a number of factors [10], of which we identify some below.

\section{Health workforce policies}

The Zambian health sector has shown capacity for HRH innovation. Examples are initiatives such as upgrading the level of training (new degree courses launched or projected, e.g. BSc Nursing), facilitating direct access to diploma level specialist training (e.g. clinical officer, psychiatry, midwifery and mental health nursing), creating new cadres to formalize task delegation from higher level cadres (e.g. dispensers, counsellors and licenciates), informal task shifting (in early 2001, the Zambian law was amended to authorize nurses to prescribe and to insert drips [11]). There were efforts to identify tasks required to meet needs and to adapt training programs to include them; an example is that of training clinical care specialists, who are physicians who receive further training to assume clinical management functions and to provide hands-on supervision to front line workers (Director HRH Administration, $\mathrm{MoH}$, personal communication, May 2008). However some of the new occupations are not recognised by professional councils, e.g. dressers, care givers, psychosocial counsellors, dispensers, medical technologists. Direct entry to advanced training reduces the back-to-school attrition associated with the loss of personnel who leave their post to train and often do not return to the public sector [4]. 
Although Zambia trains generalist cadres with internationally recognized degrees (e.g. doctors and registered nurses), other cadres are only recognized locally or regionally (e.g. Zambia enrolled nurses, clinical officers, clinical licentiates). An example of the policy of training cadres only recognized locally or regionally is that of clinical licenciates. In 2002, the MoH initiated a two-year programme of retraining clinical officers with three years of experience or more, to the level of clinical licentiates, capacitating them with surgical and obstetric skills, and more advanced skills in paediatrics and internal medicine. This training prepares them for operating autonomously in rural hospitals or in large health centres where there are no doctors. They spend six months at the Faculty, do clinical training for 20 months, and then return for 1 month to write exams. After completion, they serve a 1 year internship. These locally recognised cadres are either substitutes or assistants to other cadres; there is a long tradition of these in Zambia that predates independence (Head, Department of Community Medicine, personal communication May 2008). They are a sort of insurance against the loss of medical skills to emigration. The same reasoning applies to two categories of nurses: Registered professional nurses, internationally recognised, have higher emigration rates than enrolled nurses, who are only locally recognized [12].

In spite of these innovations, there is a shortage of skilled nurses who assume roles not only as providers of nursing care, but also as substitute to other health workers. This creates tensions which do not always help to achieve the best mix of nursing cadres and leads to misunderstandings that lead to the failure and lack of continuity of many training initiatives, such as the two year training of Zambia enrolled nurses or the six month training of nurse assistants, which were proposed by the $\mathrm{MoH}$ but opposed by the Union (President of Zambian Union of Nursing, personal communication May 2008).

\section{Emigration}

The emigration of physicians and nurses is part of the general emigration of qualified workers, which in the early 2000 's was estimated at $10 \%$ of qualified workers in Zambia, $16 \%$ in Tanzania, 26\% in Angola, 36\% in Madagascar, $42 \%$ in Mozambique, and $48 \%$ in Mauritius. For nurses, the rate was $9.2 \%$ in Zambia, 12\% in Angola, 17\% in Malawi, $19 \%$ in Mozambique 24\% in Zimbabwe, $28 \% \%$ in Madagascar, and 63\% in Mauritius. It is for physicians that the rate of emigration is particularly high: 57\% in Zambia's, surpassed only by Malawi (59\%), Angola (71\%) and Mozambique (75\%) [13].

The driving forces for migration to other countries and exit of the public sector are many: low remuneration, poor working conditions, absence of career development mechanisms, civil strife and political instability, fear to contract diseases such as HIV/AIDS and policies that encourage labour export like in the Philippines [14]. Some of these factors are present in Zambia, and may explain why out of 1,200 doctors trained in Zambia since the late 1960s, only 391 are still practicing in the Zambian public sector, a decrease that cannot be explained by normal attrition resulting from retirement or death [15]. A 2006 survey of 50 health staff in Lusaka province identified different reasons for potential migration. Low salaries are an important factor driving nurses and clinical officers to look for better paying jobs outside the public sector. Salary had less importance for doctors than inadequate diagnostic equipment and supplies. Work overload and long working hours due to shortage of health staff were also identified as push factors [15]

Emigration of health workers from Zambia is partly financed by Zambia's policy of offering Voluntary Separation Packages: these are early retirement lump sum payments promoted by the government, which are used towards migration costs [12].

\section{Working conditions and HRH management}

Working conditions are important for motivating health workers to be productive and to meet quality standards. Huddart et al [11] reported survey results showing that $100 \%$ of doctors, $80 \%$ of nurses and $92 \%$ of clinical officers wanted improvements in the cleanliness and maintenance of public health facilities. All categories of staff identified poor management of human resources as a contributory factor to issues of leave, accommodation and communication not being appropriately dealt with. This probably contributes to the high attrition rates of health personnel observed in the Zambian health sector.

This situation can be changed without significant costs as staff in the Zambian public health sector respond positively to performance-based awards [16]. Staff motivation improves substantially with even small gestures of support and encouragement from district supervisors. For example, non-financial awards are as motivating, if not more, than financial awards and do not generate as much conflict, suspicion, or frustration. Staff also feels encouraged by knowing that their performance is monitored and that this served to target support responding to their actual needs. District managers suggest that a performance-based award program, linked to the district performance management system, helps to guide them in their work, provides direction for supervisory visits and assists in monitoring health facility and district performance [16]. Many health workers generate complementary income by other activities; this is more common in urban than in rural settings (32\% and $9 \%$ of staff respectively) [17]. Besides the amount of income, other considerations are important, namely the issue of regularity of payment. In the Zambian public sector up to $15 \%$ of staff do not always 
receive their salary and $80 \%$ complained of late payments; and $10 \%$ of staff had to pay a so-called expediter fee to obtain their salaries [17].

\section{Attrition rates}

Attrition rates are also an important cause of health workforce imbalances [4]. In 2003, physicians had the highest attrition rate (9.8\%) followed by nurses (5.3\%), pharmacists (4.6\%) and laboratory technicians (3.5\%) [2]; there are differences in urban and rural attrition rates, leading to net losses of staff in rural areas (Eastern, Luapula, Northern, and Western provinces) and net staff gains in the most urbanized areas (Copperbelt and Lusaka provinces) [17]. Reasons for health personnel attrition include death (38\%), resignation $(32 \%)$, dismissal (12\%), retirement $(10 \%)$ or end of contract (8\%) [5]. Feeley et al (2004) [18] estimated the cost to the public healthcare system of personnel losses due to HIV/AIDS. If transfers to other public sector institutions are excluded, the annual loss of health professionals at the sites studied was 217 out of 2,333 filled positions, an annual attrition rate of $9 \%$. Excluding transfers, losses accounted for $21 \%$ of physician vacancies, $14 \%$ of clinical officer vacancies and $16 \%$ of nurse vacancies. In the 12 months ending in October 2003, deaths represented a significant proportion of all terminations (over $20 \%$ ); death rates were $0.4 \%$ for doctors, $2.8 \%$ for clinical officers and $3.5 \%$ for nurses working at the study sites. For doctors, mortality is not the main reason for attrition, nor the most important factor contributing for the high vacancy rates. But for clinical officers and nurses, death is the single largest reason for loss. The average age at death for all health professionals was 37.7 years. The graduation of clinical officers would have to increase $80 \%$ and of nurses $50 \%$ to offset observed mortality. In the final year of employment, those who were absent because of illness recorded an average of 28 additional days of leave. Costs associated with this additional leave, plus death or retirement related payments averaged $\$ 4,056$ for a doctor, $\$ 2,678$ for a clinical officer and $\$ 3,674$ for a nurse. In the short to medium term, the reduction of attrition and vacancies must take into account the effective treatment of AIDS, which is an important cause of absenteeism, burnout, illness and death [19-22].

\section{Health services system factors}

Over the years, there has been a steady growth of the private healthcare sector, resulting into various forms of private-public partnerships, which include the sharing of medical equipment and technologies, referral of patients, human resources and facilities.

Right-sizing of the public sector facilities and the gradual increase of "for-profit" and "not-for-profit" private health service providers presents significant policy implications in terms of their involvement in the delivery of public health services. The private sector is a major source of drain of health workers from the public sector, particularly of laboratory personnel, pharmacists and doctors (Director $\mathrm{HRH}$ Administration, $\mathrm{MoH}$, personal communication, May2008). Private salaries are more than double government ones for physicians, triple for laboratory technicians, and one third higher for midwives. NGOs are paying between $23 \%$ and $46 \%$ more than the government [23].

With the exception of the Churches Health Association of Zambia (CHAZ), other private sector participation in health service delivery in Zambia has been modest. CHAZ is an umbrella Christian NGO that supports 135 member institutions (hospitals, rural health centres, and community-based initiatives). It complements government efforts in the delivery of health care. A memorandum of understanding states that the $\mathrm{MoH}$ is mandated to provide CHAZ with trained HRH, and to pay their salary. The $\mathrm{MoH}$ deploys the staff, through the provincial health authorities. Issues of planning and performance assessments are dealt with the District Health Offices. The MoH also provides operational funds according to the number of beds. CHAZ provides infrastructure (according to the prototypes defined by the Government), complements Government efforts in logistics, provides expatriate HRH, and assumes the management of operations according to Government standards (Director HRH, CHAZ, personal communication, May.2008)

\section{Conclusion}

Factors associated with health workers shortages and with imbalances in their distribution are many and reflect local and global factors that together constitute a trap that perpetuates the situation. No single measure will correct this state of affairs [5]. Measures taken must be multifaceted, and not only sector wide, but also society wide, and supported by national and international stakeholders.

Strategies to address human resources shortages like those described here, as well as unmet needs as there exist in most African countries, have centred around staff retention through incentives such as allowances, salary top-ups, and better working conditions [24], training and retraining, including shifting as many tasks as possible away from doctors, nurses and pharmacists to non-clinical staff, enabling clinical staff to concentrate on the most complex of their specific areas of expertise [25]. These strategies are in line with the 2010 WHO recommendations on increasing access to health workers in underserved areas [26]. Scaling-up the production of health workers remains a priority, but it is expensive [5,27]. The costs of addressing shortages and deficits could reduce if rates of retention, graduation and public sector entry came closer to $100 \%$ [5]. Redistributing tasks among health worker teams through broader delegation (task-shifting), can increase technical efficiency, while maintaining quality, and thereby 
improve access and affordability [28]. A projection of enrolment needs up to 2018 concluded that even by training more qualified staff, Zambia would still face shortages, if no other efficiency enhancing measures were taken [4]. The review of the distribution of tasks among the various health cadres is a context-specific process, because it has to be locally relevant and sensitive to potential resistance; actors such as professional councils need to be brought in into the process to make task-shifting acceptable. The demonstration of regional variations in the distribution of the health workforce is a strong argument in favour of discussing options to improve access to services, including through a strategy of task-shifting and creation of new cadres. Whose tasks will be transferred to or shared with whom has to be negotiated and decided locally, within the context of a policy aimed at improving access to health services and to reducing unmet needs.

\section{Acknowledgements}

The field work for this research was supported by the World Bank. We further acknowledge support received from Fátima Ferrinho in different phases of the preparatory work for this article.

\section{Author details}

${ }^{1}$ International Public Health and Biostatistics Unit, CMDT, Instituto de Higiene e Medicina Tropical, Universidade Nova de Lisboa and Associação para o Desenvolvimento e Cooperação Garcia de Orta, Lisbon, Portugal. ²School of Medicine, University of Zambia, Lusaka, Zambia.

\section{Authors' contributions}

PF, SS and FG participated in the conception of the study, participated in field work, and in the writing up of the paper. SS and PF were responsible for the data analysis. GD was a consultant for the study and was involved with the writing up. All the authors read and approved the final manuscript.

\section{Competing interests}

The authors declare that they have no competing interests.

Received: 21 September 2010 Accepted: 19 December 2011 Published: 19 December 2011

\section{References}

1. Ministry of Health: National Health Strategic Plan (NHSP 2006-2010). Republic of Zambia, Lusaka; 2005.

2. Kombe G, Galaty D, Mtonga $V$, Banda P: Human resources crisis in the Zambian health system: a call for urgent action. Abt Associates Inc: Bethesda, MD; 2005.

3. Ministry of Health: Human Resources for Health Strategic Plan 2006 2010. Republic of Zambia, Lusaka; 2005.

4. Tjoa A, Kapihya M, Libetwa M, Schroder K, Scott C, Lee J, Mc Carthy E: Meeting human resources for health staffing goals by 2018: a quantitative analysis of policy options in Zambia. Hum Resour Health 2010, 8(1):15.

5. Chankova S, Sulzbach S: Zambia health services and system program. Occasional Paper Series. Human Resources for Health number I. Bethesda, MD: Health Services and Systems Program, Abt Associates Inc; 2006.

6. Ministry of Health: Health Institutions in Zambia. A Listing of Health Facilities According to Levels and Location for 2008 Republic of Zambia, Lusaka; 2008.

7. Ministry of Health: 2008 Annual health statistical bulletin. Lusaka, Zambia: Ministry of Health; 2009.

8. Public Service Management Division 2007. In Establishment Register for Ministry of Health to Support Estimates of Expenditure for the Year 2007. Volume 5. Republic of Zambia, Lusaka; 2007
9. Picazo O, Kagulura S: The state of human resources for health in Zambia: findings from the public expenditure tracking and quality of service delivery survey (PETS/QSDS), 2005/061, paper prepared for the "Human Resources for Health Research Conference". Mulungushi International Conference Center, Lusaka, Zambia 2007 [http://www.hrhresourcecenter.org/ node/1384].

10. Dussault G, Franceschini MC: "Not enough there, too many here: understanding geographical imbalances in the distribution of the health workforce". Hum Resour Health 2006, 4:12[http://www.human-resourceshealth.com/content/pdf/1478-4491-4-12.pdf].

11. Huddart J, Picazo O: The health sector human resources crisis in Africa: an issues paper. Washington DC: USAID Bureau for Africa. Office of Sustainable Development; 2003

12. Hamada N, Maben J, McPake B, Hanson K: International flow of Zambian Nurses. Hum Resour Health 2009, 7:83

13. Robinson R: The costs and benefits of health worker migration from East and South Africa (ESA): a literature review. Equinet Discussion Paper 49 2007.

14. Awases M, Gbary A, Nyoni J, Chatora R: Migration of Health Professionals in Six Countries: a synthesis Report. WHO Regional Office for Africa, Brazzaville 2004.

15. Lusale VD: Why do Zambian health workers migrate abroad? The brain drain of Zambian health workers. Bulletin von Medicus Mundi Schweiz 104 2007

16. Furth R: Zambia Pilot Study of Performance-based Incentives. Operations Research Results. Published for the U.S. Agency for International Development Agency (USAID) by the Quality Assurance Project (QAP), Initiatives Inc., Bethesda; 2007.

17. McCoy D, Bennett S, Witter S, Pond B, Baker B, Gow J, Chand S, Ensor T, McPake 12: Salaries and income of health workers in Sub-saharan Africa. The Lancet 2008, 371:675-681.

18. Feeley R, Rosen S, Fox M, Macwan'gi M, Mazimba A: The cost of HIV/AIDS among professional staff in the Zambian public health sector. Central Board of Health \& Center for International Health and Development, Boston University School of Public Health. Lusaka; 2004.

19. Dieleman M, Biemba S, Mphuka S, Sichinga-Sichali K, Sissolak D, van der Kwaak A, van der Wilt G-J: We are also dying like any other people, we are also people?: perceptions of the impact of HIV/AIDS on health workers in two districts in Zambia. Health Policy and Planning 2007, 22:139-148.

20. Uebel KE, Nash J, Avalos A: Caring for the caregivers: models of HIV/AIDS care and treatment provision for health care workers in Southern Africa. J Infect Dis 2007, 196(Suppl 3):S500-4.

21. World Health Organization: Treat, train, retain. The AIDS and health workforce plan. Report on the consultation on AIDS and human resources for health WHO, Geneva; 2006

22. Tawfik $L$, Kinoti SN: The impact of HIV/AIDS in the health workforce in developing countries. Background paper prepared for The World Health Report 2006 - working together for health WHO, Geneva; 2006.

23. Huddart J, Furth R, Lyons J: The Zambia HIV/AIDS Workforce Study: Preparing for Scale-up. Quality Assurance Project, Initiatives Inc., Bethesda; 2004.

24. Koot J, Martineau T: Mid Term Review. Zambian Health Workers Retention Scheme (ZHWRS) 2003 - 2004 Chapel Hill: HRH Global Resource Centre; 2005.

25. Van Damme W, Kober K, Kegels G: Scaling-up antiretroviral treatment in Southern 23 African countries with human resource shortage: How will health systems adapt? Social Science \& Medicine 2008, 66:2108-2121.

26. World Health Organization: Increasing Access to Health Workers in Remote and Rural Areas through Improved Retention: Global Policy Recommendations. WHO, Geneva; 2010 [http://www.who.int/hrh/retention/ guidelines/en/index.html].

27. Tyrell A, Russo G, Dussault G, Ferrinho P: Costing the scaling-up of Human resources for health: lessons from Guinea Bissau and Mozambique. Hum Resour Health 2010, 8:14.

28. Callaghan M, Ford N, Schneider H: A systematic review of task shifting for HIV treatment and care in Africa. Hum Resour Health 2010, 8(1):8.

doi:10.1186/1478-4491-9-30

Cite this article as: Ferrinho et al:: The human resource for health situation in Zambia: deficit and maldistribution. Human Resources for Health 2011 9:30. 